\title{
RELATIONS BETWEEN CHAIN RECURRENT POINTS AND TURNING POINTS ON THE INTERVAL
}

\author{
SHIHAI LI
}

(Communicated by Kenneth R. Meyer)

\begin{abstract}
If a point is in the $\omega$-limit set and the $\alpha$-limit set of the same point, then we call it a $\gamma$-limit point. Then a $\gamma$-limit point is an $\omega$-limit point and thus a nonwandering point. In this paper, we prove that, on the interval, a nonwandering point which is not a $\gamma$-limit point is in the closure of the set of forward images of turning points, and such points are not always the forward images of turning points. But a nonwandering point which is not an $\omega$-limit point forward image of some turning point. Two examples are given. One shows that a chain recurrent point which is not nonwandering, a $\gamma$-limit point which is not recurrent and a recurrent point which is not periodic need not be in the closure of forward images of turning points. The other shows that an $\omega$-limit point which is not a $\gamma$-limit point can be a limit point of forward images of turning points but not a forward image nor an $\omega$-limit point of any turning point.
\end{abstract}

Let $I=[0,1]$ be a compact interval on the real line. Let $f: I \rightarrow I$ be a continuous map. For each $x \in I$, we let $\operatorname{Orb}(x)=\left\{y \in I: y=f^{i}(x), i>0\right\}$. Let $T=\{x \in I: f$ is not a local homeomorphism at $x\}$ denote the set of turning points of $f$ and $\operatorname{Orb}(T)=\left\{y: y=f^{i}(x), i>0, x \in T\right\}$ denote the set of forward images of turning points of $f$. A point $x$ is called a periodic point if $f^{n}(x)=x$ for some $n>0$. A point $x \in I$ is called an $\omega$-limit $(\alpha$ limit) point if there is a $y \in I$ such that $x$ is an accumulation point of $\left\{f^{n}(y)\right\}$ (there is a sequence of integers $\left\{n_{i}\right\}$ with $n_{i} \rightarrow \infty$ and a sequence of points $\left\{y_{n_{i}}\right\}$ with $f^{n_{i}}\left(y_{n_{i}}\right)=y$ such that $\left.x=\lim _{i \rightarrow \infty} y_{n_{i}}\right)$. Denote the set of $\omega$-limit points ( $\alpha$-limit points) of $x$ by $\omega(x)(\alpha(x))$. If $x \in \omega(y) \cap \alpha(y)$ for some $y$, then $x$ is called a $\gamma$-limit point. A point $x$ is called a chain recurrent point if for any number $\varepsilon>0$ there exists a sequence of points, $x_{0}=x, x_{1}, \ldots, x_{n-1}$, $x_{n}=x$, such that $\left|f\left(x_{i}\right)-x_{i+1}\right|<\varepsilon$ for $i=0, \ldots, n-1 . C R(f), \Omega(f)$, $\Lambda(f), \Gamma(f), R(f), P(f)$ represent the collection of the chain recurrent points, nonwandering points, $\omega$-limit points, $\gamma$-limit points, recurrent points, periodic points, respectively. Without causing confusion, we write $C R, \Omega, \Lambda, \Gamma, R$, $P$ simply. Since Xiong has proved that $\Lambda^{2}(f)=\Gamma(f)$ for any continuous map on the interval in [X1], where $\Lambda^{2}(f)=\Lambda\left(\left.f\right|_{\Lambda}\right)$, we will abuse $\Lambda^{2}(f)$ and $\Gamma(f)$

Received by the editors September 30, 1990 and, in revised form, November 2, 1990.

1980 Mathematics Subject Classification (1985 Revision). Primary 58F20; Secondary 26A18.

Key words and phrases. Chain recurrent point, nonwandering point, recurrent point, turning point, $\omega$-limit point, $\alpha$-limit point, $\gamma$-limit point. 
in this paper. Sarkovskii [S] has shown that $\Lambda$ is closed. Thus $\bar{P} \subset \Lambda$. In [X1], Xiong shows that $R \subset \Gamma \subset \bar{P}$. It is easy to see that $\Lambda \subset \Omega \subset C R$. Therefore

$$
P \subset R \subset \Gamma=\Lambda^{2} \subset \bar{P} \subset \Lambda \subset \Omega \subset C R .
$$

In this paper we will determine which classes of chain recurrent points (as determined by $(*)$ ) must be contained in $\overline{\operatorname{Orb}(T)}$. By Example 2, we can see that points in $P$ and $C R \backslash \Omega$ may not be contained in $\overline{\operatorname{Orb}(T)}$. Thus we need only consider $\Omega \backslash P$.

For any continuous mapping $f: I \rightarrow I$ we have the following theorems.

Theorem 1. $\Omega(f) \backslash \Gamma(f) \subset \overline{\mathrm{Orb}(T)}$.

Theorem 2. $\Omega(f) \backslash \Lambda(f) \subset \operatorname{Orb}(T)$.

For piecewise monotone maps, Nitecki [N] has proved that $\Lambda \subset \bar{P}$. Then $\Lambda=\bar{P}$. Xiong [X2] has furthermore proved that for any point $x \in \bar{P}$ there is a point $y \in \bar{P}$ such that $x \in \omega(y)$. That is, $\bar{P}=\Lambda\left(\left.f\right|_{\bar{P}}\right)$. In [X1], Xiong proves that $\Gamma=\Lambda\left(\left.f\right|_{\bar{P}}\right)=\Lambda\left(\left.f\right|_{\Lambda}\right)=\Lambda^{2}$ for any continuous map $f$. Thus $\Lambda=\bar{P}=\Gamma=\Lambda^{2}$ for piecewise monotone maps, and $\Omega \backslash \Gamma \subset \operatorname{Orb}(T)$. But this fact cannot be generalized to any continuous maps. We will point out in Example 1 that the Example 1 in $[\mathrm{BC}]$ is a counterexample; in fact, there is a point $x \in \Lambda \backslash \Gamma$ with $x \notin \operatorname{Orb}(T)$. We also point out that the point $x \notin \bigcup_{t \in T} \omega(t)$.

In Example 2, we will find out that $\Gamma \backslash P$ need not be a subset of $\overline{\operatorname{Orb}(T)}$. Xiong [X2] has proved that if $f$ has no periodic points of periods not a power of 2 and $f$ is piecewise monotone, then any point in $R \backslash P$ is an $\omega$-limit point of some turning point. If we check the proof of this property in [X2] carefully, we can see that $R \backslash P \subset \overline{\mathrm{Orb}(T)}$ for any continuous map with no periodic points of period not the power of 2 . This fact is not true if $f$ has a periodic point with period not the power of 2 . Example 2 is a counterexample.

Without mention, $f$ is always a continuous mapping of $I$ to itself.

\section{Proof OF TheOREM 1}

The following lemmas are known.

Lemma 1 ([CN]). $x \in \Omega$ if and only if $x \in \alpha(x)$.

Lemma 2. If $(f(x)-x)(f(y)-y)<0$, then there exists a fixed point between $x$ and $y$.

It is easy to prove the following lemma.

Lemma 3. $T\left(f^{n}\right) \subset \bigcup_{i=0}^{n-1} f^{-i}(T(f))$ (therefore, if $q \in T\left(f^{n}\right)$, then there is a point $q^{\prime} \in T(f)$ such that $\left.f^{n}(q) \in \operatorname{Orb}\left(q^{\prime}\right)\right)$.

Proof of Theorem 1. Suppose that $x \in \Omega \backslash \bar{P}$ and $C$ is the connected component of $I \backslash \bar{P}$ which contains $x$. Obviously, $x \notin R$ since $\bar{P}=\bar{R} \supset R$ [CH, Y]. Then, there exist two points $a, b \in C$ such that $(a, b) \cap \operatorname{Orb}(x)=\varnothing$.

By Lemma 1, without loss of generality, there exist a sequence of points $y_{i} \in(a, x)$ and a sequence of positive integers $n_{i} \rightarrow \infty$ such that $f^{n_{i}}\left(y_{i}\right)=x$ and $y_{i} \rightarrow x$ as $i \rightarrow \infty$. Obviously, we could suppose that $y_{i}<y_{i+1}$ and $n_{i}<n_{i+1}$. 
Since $f^{n_{i}}\left(y_{i}\right)=x>y_{i}$ and $(a, b) \cap P=\varnothing$, it follows that $f^{n_{i}}\left(y_{i-1}\right)>$ $y_{i-1}$ by Lemma 2. Also $f^{n_{i}}\left(y_{i-1}\right)=f^{n_{i}-n_{i-1}}\left(f^{n_{i-1}}\left(y_{i-1}\right)\right)=f^{n_{i}-n_{i-1}}(x)$. Since $(a, b) \cap \operatorname{Orb}(x)=\varnothing$, it follows that $f^{n_{i}}\left(y_{i-1}\right) \geq b$. By the same reason, $f^{n_{i}}(x) \geq b$.

Since $f^{n_{i}}\left(y_{i}\right)=x<b$, there must be a turning point $q \in\left(y_{i-1}, x\right)$ of $f^{n_{i}}$ with $q<f^{n_{i}}(q) \geq x$. This is to say that $f^{n_{i}}(q) \in\left(y_{i-1}, x\right]$. By Lemma 3, there exists a point $q^{\prime} \in T(f)$ such that $f^{n_{i}}(q) \in \operatorname{Orb}\left(q^{\prime}\right)$.

Note that $q$ is a function of $i$. Since $f^{n_{i}}(q) \rightarrow x$ as $i \rightarrow \infty$ it follows that $x \in \overline{\operatorname{Orb}(T(f))}$.

Thus $\Omega \backslash \bar{P} \subset \overline{\mathrm{Orb}(T(f))}$. Note that the proof of Theorem 1 in [X2] implies that $\bar{P} \backslash \Gamma \subset \overline{\operatorname{Orb}(T(f))}$. Therefore we have proved the theorem.

\section{Proof of Theorem 2}

Lemma 4. If $n_{i}$ are natural numbers and $K_{i}(i=1,2, \ldots)$ are closed intervals such that $K_{i+1} \subset K_{i}$ and $f^{n_{i}}\left(K_{i}\right) \supset K_{i+1}$ for all $i \geq 1$, and $\bigcap_{i=1}^{\infty} K_{i}$ consists of a single point $x$, then there exists a point $y \in K_{1}$ such that $f^{l_{j}}(y) \in K_{j+1}$ and $f^{l_{j}}(y) \rightarrow x$ as $j \rightarrow \infty$ with $l_{j}=\sum_{i=1}^{j} n_{i}$.

Proof. By assumption, we have $f^{l_{i}}\left(K_{1}\right) \supset K_{i+1}$ for all $i \geq 1$. We can form a sequence of closed intervals $\left\{K_{j}^{\prime}\right\}$ such that $K_{1}^{\prime} \subset K_{1}, K_{j+1}^{\prime} \subset K_{j}^{\prime}$, and $f^{l_{j}}\left(K_{j}^{\prime}\right)=K_{j+1}$ for each positive integer $j$. It follows that $f^{l_{i}}\left(K_{j}^{\prime}\right) \subset K_{i+1}$ for all $i<j$. Let $y \in \bigcap_{j=1}^{\infty} K_{j}^{\prime}$. Then $f^{l_{j}}(y) \in K_{j+1}$ for all $j \geq 1$ and furthermore $f^{l_{j}}(y) \rightarrow x$.

If $C$ is a component of $I \backslash \bar{P}$, it has been shown [X4, C] that for any $x$, $y \in C$,

$$
\left(f^{n}(x)-x\right)\left(f^{m}(y)-y\right)>0
$$

for all integers $n, m>0$ such that $f^{n}(x), f^{m}(y) \in C$.

If $f^{n}(x)>x$ whenever $f^{n}(x) \in C$ (or $f^{n}(x)<x$ ), we say that $C$ is of increasing type (or decreasing type).

Lemma 5. If $x \notin \Lambda$ is in a component $C$ of $I \backslash \bar{P}$ of increasing type, then there is an interval $[z, x]$ such that, for any $y \in[z, x]$ and any positive integer $n$, $f^{n}(y) \notin[z, x)$.

Proof. Suppose it is not true. Then there is an increasing sequence of points $y_{i} \in C$ with $y_{i} \neq x$ and $y_{i} \rightarrow x$, and a sequence of integers $n_{i}>0$ such that $y_{i}<f^{n_{i}}\left(y_{i}\right)<y_{i+1}<x$. Since $f^{n_{i}}\left(y_{i}\right)>y_{i}$ and there is no periodic point in $\left[y_{i}, x\right]$, we know that $f^{n_{i}}(x)>x$ by Lemma 2 .

Denote $K_{i}=\left[y_{i}, x\right]$. Then

$$
f^{n_{i}}\left(K_{i}\right) \supset\left[f^{n_{i}}\left(y_{i}\right), f^{n_{i}}(x)\right] \supset K_{i+1} .
$$

Also, $\bigcap_{i=1}^{\infty} K_{i}$ consists of a single point $x$. By Lemma 4, there is a point $y \in K_{1}$, so that $f^{l_{j}}(y) \rightarrow x$ where $l_{j}=\sum_{i=1}^{j} n_{i}$. It follows that $x \in \omega(y) \subset \Lambda$. This contradicts the assumption that $x \notin \Lambda$.

Proof of Theorem 2. Let $x \in \Omega \backslash \Lambda$. Recall that $\Lambda$ is closed. Since $x \notin \Lambda$ and $\Lambda \supset R$, we have $x \notin R$. Then there is an interval $(a, b)$ containing no points of $\Lambda$ such that $x \in(a, b)$ and $(a, b) \cap \operatorname{Orb}(x)=\varnothing$. Also, as 
in the proof of Theorem 1 , for any $x \in \Omega \backslash \Lambda$ we may assume without loss of generality that there exist a sequence of points $y_{i}<x$ in $(a, b)$ and an increasing sequence of positive integers $n_{i} \rightarrow \infty$ such that $f^{n_{i}}\left(y_{i}\right)=x$, for all $i=1,2, \ldots$ and $y_{i} \rightarrow x$. Then $(a, b)$ is of increasing type. By Lemma 5 , there is an interval $[z, x]$ so that, for any $y \in[z, x]$ and any integer $n>0$, we have $f^{n}(y) \notin[z, x)$. Without loss of generality, we can assume that $y_{1}, y_{2}, \ldots, y_{i}, \ldots$ are all in $[z, x]$. Then $f^{n}\left(y_{i}\right) \notin[z, x)$.

By Lemma 2, for any point $w$ in $\left(y_{1}, y_{3}\right)$, we have $f^{n_{2}}(w) \geq x$ since $f^{n_{2}}\left(y_{2}\right)=x$. Thus $y_{2}$ is a turning point of $f^{n_{2}}$. By Lemma 3, $x=f^{n_{2}}\left(y_{2}\right) \in$ $\operatorname{Orb}(T(f))$.

\section{EXAMPLES}

Proposition 6 [X3]. $\overline{R(f)}=R(f)$ implies that $f$ has only periodic points of periods $2^{n}, n \geq 0$.

In Example 1 we show that $\Lambda \backslash \bar{P}$ is not always contained in $\operatorname{Orb}(T)$.

Example $1[\mathrm{BC}]$. Let $f:[-1,2] \rightarrow[-1,2]$ be the map constructed by Block and Coven in Example 1 of [BC]. The following properties are either already observed in $[\mathrm{BC}]$ or easy to be seen.

(1) The Cantor middle third set $C$ in $[0,1]$ is a minimal set under $f$.

(2) $\omega\left(-\frac{2}{3}\right)=C$.

(3) $-\frac{2}{3} \in \Lambda \backslash \bar{P}$.

(4) Any point $t \in\left[-\frac{2}{3}, 0\right]$ is a turning point and $f(t)=\frac{2}{3} \in C .1$ is a turning point and $1 \in C$. Let $s_{n}=\frac{3^{n}-1}{3^{n}}$ for integers $n \geq 1$. Then each $s_{n}$ is a turning point and $s_{n} \in C$.

(5) Except for those turning points described in (4), all other turning points can be represented by $t_{n}=1-\frac{1}{2 \times 3^{n}}$ for $n \geq 0$. Moreover $f^{m_{n}}\left(t_{n}\right)=$ $-\frac{2}{3}-\frac{1}{3^{n+2}}$ for some integer $m_{n}>0$ and $f^{2}\left(-\frac{2}{3}-\frac{1}{3^{n}}\right)=\frac{2}{9}+\frac{1}{3^{n+1}} \in C$.

By (5) we know that $-\frac{2}{3} \in \overline{\operatorname{Orb}(T)}$. $-\frac{2}{3}$ can never be the image of a turning point in (4) since $f(C) \subset C$ by (1). For the $t_{n}$ 's in (5), we know that for $0<j<m_{n}$ we have $f^{j}\left(t_{n}\right) \in[0,2]$. Also, $f^{m_{n}}\left(t_{n}\right)=-\frac{2}{3}-\frac{1}{3^{n+2}}, f^{m_{n}+1}\left(t_{n}\right) \in$ $\left[\frac{1}{3}, \frac{2}{3}\right]$, and $f^{m_{n}+2}\left(t_{n}\right) \in C$. Thus $-\frac{2}{3} \notin \operatorname{Orb}\left(t_{n}\right)$. Therefore $-\frac{2}{3} \notin \operatorname{Orb}(T)$. By $(3),-\frac{2}{3} \in(\Lambda \backslash \bar{P}) \cap(\overline{\operatorname{Orb}(T)} \backslash \operatorname{Orb}(T))$.

Moreover by (1) and the above argument it is easy to see that $\omega(t) \subset C$ for any turning point $t \in T$. Thus $-\frac{2}{3} \notin \bigcup_{t \in T} \omega(t)$.

Example 2. (See Figure 1) Let $f:[0,3] \rightarrow[0,3]$ be defined as follows.

$$
f(x)= \begin{cases}\sqrt{x}, & \text { when } x \in[0,1], \\ 4 x-3, & \text { when } x \in\left[1, \frac{3}{2}\right], \\ -2 x+6, & \text { when } \left.x \in \frac{3}{2}, 3\right]\end{cases}
$$

For any point $x \in(0,1)$ we have that $1 \in \alpha(x) \cap \omega(x)$. Note that 1 is a fixed point. So $x \in C R$. But $x \notin \Omega$. Thus $x \in C R \backslash \Omega$. Obviously $x \notin \overline{\operatorname{Orb}(T)}$. Next we show that $\Gamma \backslash R$ may not be contained in $\overline{\operatorname{Orb}(T)}$. 


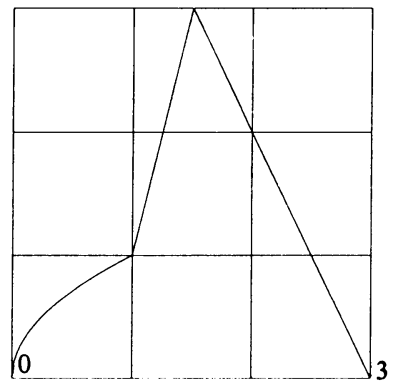

FIGURE 1. $P \not \subset \overline{\operatorname{Orb}(T)} ; C R \backslash \Omega \not \subset \overline{\operatorname{Orb}(T)} ;(\Gamma \backslash R) \cap$ $\overline{\operatorname{Orb}(T)}=\varnothing$.

The turning point of $f$ is $\frac{3}{2}$. $\operatorname{Orb}\left(\left\{\frac{3}{2}\right\}\right)=\left\{0, \frac{3}{2}, 3\right\}$. Since $\frac{3}{2}$ and 3 are wandering points and 0 is a fixed point, we have $\Omega=\Gamma$ by Theorem 1. Moreover, $0, \frac{3}{2}$, and 3 are not in the set $\Gamma \backslash R$, i.e., $(\Gamma \backslash R) \cap \overline{\operatorname{Orb}(T)}=\varnothing$. It is easy to see that $f$ has a periodic point with period 3. Since $\bar{R} \subset \Omega=\Gamma$ it follows from Proposition 6 that $\Gamma \backslash R \neq \varnothing$.

Moreover $R \neq P$. For otherwise, $\bar{P}=P$ by [X5], a contradiction to $\Gamma \backslash R \neq$ $\varnothing$. Evidently $\frac{3}{2}$ and 3 are not recurrent points. Thus $(R \backslash P) \cap \overline{\operatorname{Orb}(T)}=\varnothing$. Without mention, $f$ is always a continuous mapping of $I$ to itself.

\section{ACKNOWLEDGMENT}

This work was done under the guidance of Professor Block. Without his help, it would not have been done. I would like to thank him very much.

\section{REFERENCES}

[B] L. Block, Continuous maps of the interval with finite nonwandering set, Trans. Amer. Math. Soc. 240 (1978), 221-230.

[BC] L. Block and E. M. Coven, $\omega$-limit sets for maps of the interval, Ergodic Theory Dynamical Systems 6 (1986), 335-344.

[C] W. A. Coppel, Continuous maps of an interval, Lecture Notes in Australian National University, 1984.

[CH] E. M. Coven and G. H. Hedlund, $\bar{P}=\bar{R}$ for maps of the interval, Proc. Amer. Math. Soc. 79 (1980), 316-318.

[CN] E. M. Coven and N. Nitecki, Nonwandering sets of the powers of maps of the interval Ergodic Theory and Dynamical Systems 1 (1981), 9-13.

[N] Z. Nitecki, Periodic and limit orbits and the depth of the centre for piecewise monotone interval maps, Proc. Amer. Math. Soc. 80 (1980), 511-514.

[S] A. N. Sarkovskii, On some properties of discrete dynamical systems, Proc. Internat. Colloq. on Iteration Theory and its Appl. (Toulouse, 1982), Univ. Paul Sabatier, pp. 153-158.

[X1] J.-C. Xiong, The attracting center of a continuous self-map of the interval, Ergodic Theory Dynamical Systems 8 (1988), 205-213.

[X2] - The closure of periodic points of a piecewise monotone map of the interval, preprint.

[X3] _ The perioids of periodic points of continuous self-maps of the interval whose recurrent points form a closed set, J. China Univ. Sci. Tech. 13 (1983), 134-135.

[X4] _,$\Omega\left(\left.f\right|_{\Omega(f)}\right)=\overline{P(f)}$ for every continuous self map $f$ of the interval, Kexue Tongbao (English version) 28 (1983), 21-23. 
[X5] _ Set of almost periodic points of a continuous self-map of an interval, Acta Math. Sinica (N.S.) 2 (1986), 73-77.

[Y] L. Young, A closing lemma on the interval, Invent. Math. 54 (1979), 179-187.

Department of Mathematics, University of Florida, Gainesville, Florida 32611 E-mail address: shi@math.ufl.edu 